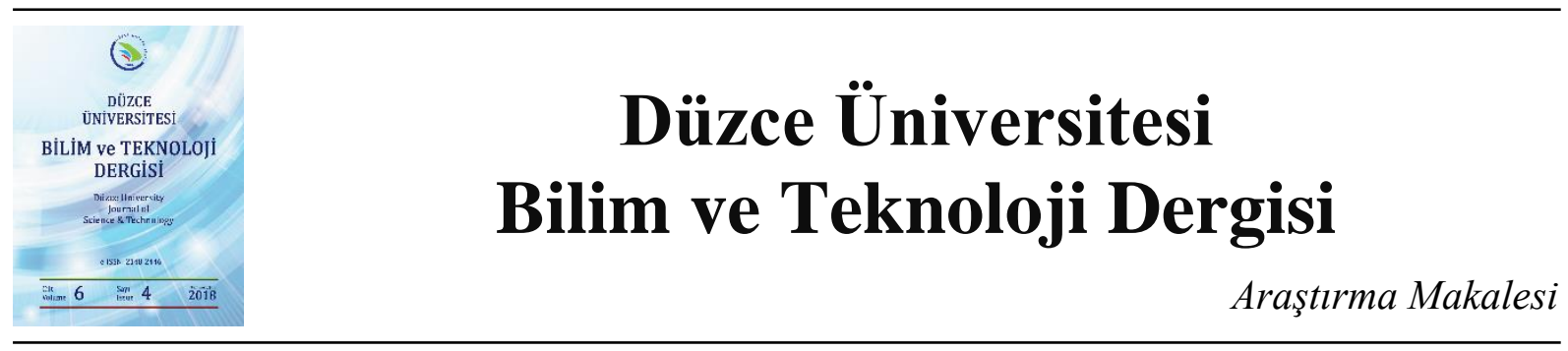

\section{Kolektif Öğrenmeye Dayalı Çok Değişkenli Desen Analizinin Klinik Karar Destek Sistemlerinde Uygulanması}

\author{
Ayşe DEMIRHAN ${ }^{\mathrm{a},{ }^{*}}$ \\ ${ }^{a}$ Elektrik-Elektronik Mühendisliği Bölümü, Teknoloji Fakültesi, Gazi Üniversitesi, Ankara, TÜRKiYE \\ ** Sorumlu yazarın e-posta adresi: ayseoguz@gazi.edu.tr
}

\begin{abstract}
ÖZET
Çok değişkenli desen analizi (ÇDDA) klinik verilerin incelenmesi için yaygın olarak kullanılan güçlü bir tekniktir. ÇDDA uygulamalarında genellikle makine öğrenmesi yöntemleri kullanılmaktadır. Kolektif öğrenme algoritmaları çok sayıda makine öğrenmesi metodunu bir araya getirilerek sınıflama performansını arttırmaktadır. Kadınlarda en sık görülen ikinci kanser türü olan serviks kanseri düşük gelirli ülkelerde önemli bir ölüm nedenidir. İlk evrelerinde belirti göstermeyen serviks kanseri erken teşhis edildiğinde tamamen tedavi edilebilmektedir. Bu çalışmada rastgele ormanlar, aşırı rassal ağaçlar ve Adaboost kolektif öğrenme yöntemleri kullanılarak hastaların serviks kanseri riskini çeşitli risk faktörlerinden faydalanarak farklı tarama yöntemleri açısından tahmin eden bir ÇDDA uygulaması yapılmıştır. Aşırı rassal ağaçlar algoritmasından Hinselmann, Schiller, sitoloji ve biyopsi tarama yöntemlerinin hastaların serviks kanseri olması riskini tahmin başarısı sırasıyla $\% 99, \% 96, \% 97$ ve $\% 97$ olmuştur.
\end{abstract}

Anahtar Kelimeler: Kolektif öğrenme algoritmaları, Rastgele ormanlar, Aşırı rassal ağaçlar, Adaboost, Klinik karar destek sistemleri

\section{Application of Ensemble Learning Based Multivariate Pattern Analysis On Clinical Decision Support Systems}

\begin{abstract}
Multivariable pattern analysis (MVPA) is a powerful technique that is used widely to examine clinical data. Machine learning methods are generally used in MVPA applications. Ensemble learning algorithms increase the classification performance by combining a large number of machine learning methods. Cervical cancer that is the second most common cancer type in women is a major cause of death in low-income countries. Cervical cancer, which has no symptoms in its initial stages, can be completely cured if it is diagnosed early. In this study a MVPA application is performed that uses random forests, extremely randomized trees and Adaboost ensemble learning methods to estimate the risk of cervical cancer of the patients by using the various risk factors for different
\end{abstract}

Geliş: 11/06/2018, Düzeltme: 28/07/2018, Kabul: 30/07/2018 
screening strategies. Prediction performance of the extremely randomized trees for the cervical cancer risk was 99\%, 96\%, 97\% and 97\% for the Hinselmann, Schiller, citology and biopy screening methods.

Keywords: Ensemble learning algorithms, Random forests, Extremely randomized trees, Adaboost, Clinical decision support systems

\section{GiRISS}

$\mathrm{K}$ linik karar destek sistemleri (KKDS), doktorlara hastaya ait tıbbi verileri kullanarak vereceği kararlar konusunda yardım eden bilgisayar sistemleridir. Hastaya özgü veriler ile uygulanan KKDS'nin teşhis koyma, önleyici bakım, hastalık yönetimi ve reçetelendirme konularında sağladığı destekle hasta sonuçlarını iyileştirdiği ve bakım maliyetlerini düşürdüğü yapılan çalışmalarla gösterilmiştir [1]. KKDS'nin tasarımı ve uygulamasında matematiksel modelleme, örüntü tanıma ve büyük veritabanlarının istatistiksel analizi teknikleri ile birlikte Bayes modellemesi, karar ağaçları, yapay sinir ağları gibi makine öğrenmesi algoritmaları da yaygın olarak kullanılmaktadır. Sağlık hizmetlerinde üretilen büyük hacimli veriler nedeniyle hekim uygulamalarını, hastalık yönetimini ve kaynak kullanımını iyileştirmek için veri madenciliği yöntemlerine duyulan ilgi her geçen gün artmaktadır [1,2].

$\mathrm{Bu}$ çalışmada serviks kanserinin teşhis edilmesine yönelik bir KKDS çalışması yapılmıştır. Serviks kanseri, anormal hücrelerin serviks üzerinde kontrol dışı büyümesi ile meydana gelir. Kötü huylu bir tümör olan serviks kanserinin görülme sıklığı meme kanserinden sonra ikinci sıradadır [3]. Günümüzde serviks kanserini teşhis etmek için kullanılan çok sayıda tarama ve teşhis yöntemi vardır. Örneğin, sitoloji, kolposkopi ve biyopsi tarama yöntemleri serviks lezyonlarını tespit etmek için kullanılmaktadır. Düşük gelirli ve gelişmekte olan ülkelerde kaynaklar kısıtlıdır ve hastalar genellikle düşük problem farkındalı̆̆ nedeniyle rutin taramalara bağl1lık göstermemektedir. Bu nedenle hastalığın teşhisindeki en iyi tarama yöntemini ve her hastanın risk durumunu tespit etmek büyük önem taşımaktadır [4]. Serviks kanserine yönelik geliştirilen KKDS ile hangi metabolitlerin istatistiksel öneme sahip olduğu ve hastalığın sınıflandırılmasında başarılı olduğu [3], hastaların yaşam periyotlarını etkileyen faktörlerin belirlenmesi [5] ve serviks kanserinin teşhisi ile ilişkili faktörlerin bulunması [6] konularında araştırmalar yapılmıştır.

Çok değişkenli desen analizi (ÇDDA), klinik verilerin analizi için klasik istatistik uygulamalara dayalı tek değişkenli yöntemlere göre daha güçlü bir tekniktir. Genellikle makine öğrenmesi yöntemleri ile gerçekleştirilen ÇDDA klinik teşhis uygulamalarında yaygın olarak kullanılmakta ve başarılı sonuçlar üretmektedir [7, 8]. ÇDDA algoritmaları hastalardan toplanan klinik tarama verilerinden faydalanarak geliştirilen tahmin modelleri olarak kullanılırlar. Bountris ve diğerleri [7] serviks kanserinde teşhis doğruluğunu arttırmak için klasik ve yardımcı yöntemlerin sonuçlarını birleştirdikleri ÇDDA çalışmasında yapay sinir ağlarını kullanmışlardır. Geliştirdikleri bu KKDS ile \%89,4 duyarlı1ık, \%97,1 belirlilik ve \%89,4 pozitif tahmin doğruluğuna ulaşmışlardır. Mayr ve diğerleri [9] yaptıkları çalışmada tümör morfolojisi ve mikrosirkülasyon verilerinin birlikte kullanılmasının ileri serviks kanserli hastalarda lokal yetmezlik tahmininde her bir parametrenin tek başına kullanılmasından daha doğru tahmin potansiyeline sahip olduğunu göstermişlerdir. 
Kolektif öğrenme, çok sayıda öğrenme algoritmasının belirli bir problemi çözmek üzere bir araya getirilmesidir. Buradaki amaç tek bir öğrenme algoritmasından elde edilecek tahmin doğruluğunu arttırmak ve varyansı azaltmaktır. Kolektif öğrenmenin bagging ve boosting olmak üzere iki farklı uygulama şekli vardır. Bagging yöntemi veri setindeki rastgele alt örnekler üzerinde çalışan çok sayıda öğrenme algoritmasından elde edilen bireysel tahminlerin nihai bir tahmin oluşturmak için toplanmasıdır. Boosting yöntemindeyse öğrenme algoritmaları peş peşe çalıştırılmaktadır. Öğrenme algoritmasının kullanacağı eğitim seti, bir önceki algoritmanın performansına göre hata yapılan örneklere öncelik verilerek belirlenmektedir $[10,11]$.

$\mathrm{Bu}$ çalışmada, kolektif öğrenmeye dayalı ÇDDA ile serviks kanserinin teşhisi için bir KKDS geliştirilmiştir. Hastalardan klinik olarak elde edilen 32 risk faktörü kullanılarak 4 farklı tarama yöntemi (Hinselmann, Schiller, sitoloji ve biyopsi) için kanser riski tahmin edilmiştir. Bu amaçla kolektif öğrenme algoritmalarından bagging yöntemini kullanan rastgele ormanlar ve aşırı rassal ağaçlar ile boosting yöntemini kullanan Adaboost ile sınıflama yapılmış ve elde edilen sınıflama doğrulukları karşılaştırılmıştır.

Bu çalışmanın ikinci bölümünde kullanılan veri setinin özellikleri verilmiştir. Aynı zamanda çalışmada kullanılan kolektif öğrenme algoritmaları tanıtılmıştır. Üçüncü bölüm KKDS'nin detayları ve parametreleri ile çalışmadan elde edilen deneysel sonuçlara ayrılmıştır. Dördüncü bölümde ise elde edilen sonuçlar yorumlanarak yapılan çalışmanın önemi değerlendirilmiştir.

\section{MATERYAL VE YÖNTEM}

\section{A. VERI}

Bu çalışmada Caracas Üniversitesi Hastanesi’nden toplanan serviks kanseri verileri kullanılmıştır [4]. $\mathrm{Bu}$ veri seti 858 hastaya ait demografik veriler, alışkanlıklar ve tıbbi geçmiş kayıtlarına ilişkin 32 farklı risk faktörüne ait özellikler içermektedir. Bu özellikler arasında yaş, cinsel eş sayısı, hamilelik sayısı, sigara içilip içilmediği, hormonal doğum kontrolü yöntemlerinin kullanılıp kullanılmadığı, kullanıldıysa kaç yıl devam ettiği, rahim içi cihaz kullanıp kullanılmadığı, cinsel yolla bulaşan enfeksiyon geçirilip geçirilmediği, geçirildiyse sayısı ve türü gibi hastaya ait detaylı bilgiler bulunmaktadır. Veri seti hedef değişkenler olarak serviks kanseri teşhisinde kullanılan 4 farklı tarama yöntemi açısından hastanın kanser riski taşıyıp taşımadığı bilgisini de içermektedir. Bu tarama yöntemleri Hinselmann, Schiller, sitoloji ve biyopsidir. Hinselmann yöntemi asetik asit kullanılarak, Schiller ise lügol iyodin kullanılarak yapılan kolposkopidir.

\section{B. KOLEKTIF ÖĞRENME ALGORITMALARI}

Rastgele ormanlar çok sayıda karar ağacının bir araya getirilmesi ile oluşturulan bir kolektif öğrenme yöntemidir. Rastgele orman içerisindeki her bir ağaç birbirinden bağımsız olarak giriş verisi içerisinden rastgele örneklenmiş vektörlere bağlı olarak sonuç üretir. Bu rastgele örnekleme her ağaç için eşit dağılıma sahip olacak şekilde yapılır. Ormandaki ağaçların sayısını arttırmak belirli bir noktaya kadar sistemin performansını arttırmaktadır. Rastgele bir ormanın başarımı ormanda yer alan her bir ağacın gücüne ve ağaçlar arasındaki ilişkiye bağlıdır. Ağaçtaki düğümleri ayırmak için eğitimde kullanılan özelliklerin rastgele seçilmesi hata oranını düşürür. Rastgele ormanlar giriş verisinde bulunan 
gürültülere karşı da dayanıklıdır. Tüm ağaçlardan gelen sınıflama sonuçları oylama yöntemi ile bir araya getirilir ve en çok oy olan sınıf çıkış olarak belirlenir [12].

Ekstra ağaçlar olarak da bilinen aşırı rassal ağaçlar danışmanlı sınıflama ve regresyon problemlerinde kullanılan ağaç tabanlı bir kolektif öğrenme algoritmasıdır. Aşırı rassal ağaçlardaki rassallık ağaç düğümlerinin bölünmesi sırasında rastgele ormanlarla kıyaslandığında bir adım daha ileri gider. $\mathrm{Bu}$ yöntemde ağaç dügümleri bölünürken hem öznitelik hem de eşik değeri seçiminde güçlü bir rassallık kullanılmaktadır. Rastgele ormanlardaki gibi giriş özelliklerinin rastgele bir alt kümesi kullanılır fakat düğümlerin bölünmesi sırasında en ayırt edici eşik değerini aramak yerine giriş verisindeki her özellik için rastgele bir eşik değerleri atanır. Bu rastgele oluşturulmuş eşik değerlerinin en iyisi düğümlerin bölünmesinde kullanılır. Bu uygulama yanlılı̆̆ 1 bir miktar arttırmakla birlikte modelin varyansını düşürmeyi sağlar [13].

Adaboost (Adaptive Boosting) algoritması sınıflama performansını arttırmak için zayıf öğreniciler de denilen çok sayıda öğrenme algoritmasının peş peşe çalıştırılmasıdır. Sistemin çıkışı zayıf öğrenicilerin çıkışlarının ağırlıklı ortalaması ile elde edilir. AdaBoost algoritmasında sonra gelen zayıf öğrenciler önce çalışan öğreniciler tarafından yanlış sınıflandırılan örneklere öncelik vererek uyarlanmaktadır. Algoritmanın her bir tekrarında eğitim örneklerinin her birine $w_{1}, w_{2}, \ldots w_{n}$ ağırlıkları uygulanır. Başlangıçta bu ağırlıkların hepsi $w_{i}=1 / N$ olarak ayarlanır. Böylece algoritmanın ilk tekrarında zayıf öğreniciler orijinal veri seti üzerinde çalışırlar. Algoritmanın her tekrarında örnek ağırlıkları ayrı ayrı değiştirilir ve öğrenme algoritması yeniden ağırlıklandırılmış verilere tekrar uygulanır. Bir önceki adımdaki model tarafindan yanlış tahmin edilen eğitim örnekleri ağırlıkları arttırılırken doğru tahmin edilen örneklerin ağılıkları azaltılır. Algoritma çalıştıkça tahmin edilmesi zor örneklerin ağırlıkları giderek artar. Böylece sonraki her bir zayıf öğrenci, önceki öğrenici tarafından gözden kaçırılan örneklere odaklanmaya zorlanır [14]. Bu çalışmada zayıf öğrenici olarak karar ağaçları ve sınıflama algoritması olarak orijinal Adaboost yönteminin geliştirilmiş bir versiyonu olan Adaboost-SAMME.R yöntemi kullanılmıştır [15]. Modelin güncellenmesinde sınıf etiketleri yerine ağırlıklandırılmış sınıf olasılıkların kullanan bu yöntemde zayıf öğrenicilerin performansının rastgele tahminden (1/2) daha yüksek olması gerekmektedir. Yanlış sınıflandırılmış örneklere Adaboost'a göre daha yüksek ağırlık değerleri atayan bu yöntemin ikili sınıflama problemlerinde orijinal Adaboost algoritmasına göre daha yüksek performans sergilediği gösterilmiştir [16].

\section{BULGULAR VE TARTIȘMA}

$\mathrm{Bu}$ çalışmada rastgele ormanlar, aşırı rassal ağaçlar ve Adaboost kolektif öğrenme algoritmaları kullanılarak gerçekleştirilen ÇDDA ile hastaların serviks kanseri riski tahmin edilmiştir. Tüm öğrenme algoritmaları Python scikit-learn kullanılarak gerçekleştirilmiştir [17].

Toplam 858 hastaya ait 32 özellik içeren veri setinde pek çok hasta bazı sorulara mahremiyet nedeniyle cevap vermemiştir. Bu nedenle veri setinden eksik bilgileri bulunan hastalara ait kayıtlar çıkarılmıştır. Veri setindeki sağlıklı ve kanser hastası sınıflarının dağılımları dengesizdir. Bu nedenle verilere sınıf dağılımları eşit olacak şekilde oversampling uygulanmıştır. İki adet risk faktöründe çok fazla eksik değer olduğundan bunlar veri setinden çıkarılmıştır. Sonuçta 668 hastaya ait 30 özellik ve 4 farklı hedef tarama yöntemi kullanılmıştır. 
Tüm öğrenme algoritmalarının performansı 10-kat çapraz doğrulama yöntemi ile doğruluk, duyarlılık, belirlilik ve AUC performans ölçütleri kullanılarak değerlendirilmiştir. Öğrenme algoritmalarının parametreleri deneysel olarak en yüksek başarım gösteren değerler olarak seçilmiştir.

Rastgele ormanlar algoritmasını kullanarak yapılan ikili sınıflama işleminde ormandaki ağaç sayısı 10, dügüumlerdeki bölünmenin kalitesini ölçmek içinse Eşitlik (1)'de verilen 'gini' fonksiyonu kullanılmıştır. Burada $j$ sınıf sayısı, $t$ o dügümdeki örneklerin alt kümesi, $P(i \mid t)$ ise dügüm alt kümesinden i sınıfının seçilme olasılığıdır.

$\operatorname{gini}(t)=1-\sum_{i=1}^{j} P(i \mid t)$

Rastgele ormanların oluşturulmasında en iyi düğüm ayrımını ararken kullanılacak özellik sayısı toplam özellik sayısına eşit olarak seçilmiş ve eğitim için kullanılan örneklere bootstrap işlemi uygulanmıştır. Tablo 1 rastgele orman algoritmasından 10-kat çapraz doğrulama sonucunda eğitim ve test verileri için elde edilen ortalama sınıflama sonuçlarını göstermektedir. Parantez içinde verilen rakamlar standart sapmayı belirtmektedir.

Tablo 1. Rastgele orman algoritmasından elde edilen sınıflama sonuçları

\begin{tabular}{ccccccccc}
\hline & \multicolumn{9}{c}{ Ĕ̆itim } & \multicolumn{4}{c}{ Test } \\
\hline & Doğruluk & Kesinlik & Hassasiyet & AUC & Doğruluk & Kesinlik & Hassasiyet & AUC \\
\hline \multirow{2}{*}{ Hinselmann } & 1,00 & 0,99 & 1,00 & 1,00 & 0,99 & 0,97 & 1,00 & 1,00 \\
& $( \pm 0,00)$ & $( \pm 0,00)$ & $( \pm 0,00)$ & $( \pm 0,00)$ & $( \pm 0,02)$ & $( \pm 0,03)$ & $( \pm 0,00)$ & $( \pm 0,01)$ \\
\hline \multirow{2}{*}{ Schiller } & 0,99 & 0,99 & 0,99 & 1,00 & 0,95 & 0,96 & 0,95 & 0,98 \\
& $( \pm 0,00)$ & $( \pm 0,00)$ & $( \pm 0,00)$ & $( \pm 0,00)$ & $( \pm 0,03)$ & $( \pm 0,04)$ & $( \pm 0,03)$ & $( \pm 0,02)$ \\
\hline \multirow{2}{*}{ Sitoloji } & 0,99 & 0,98 & 0,97 & 1,00 & 0,97 & 0,94 & 0,84 & 0,93 \\
& $( \pm 0,00)$ & $( \pm 0,01)$ & $( \pm 0,01)$ & $( \pm 0,00)$ & $( \pm 0,03)$ & $( \pm 0,12)$ & $( \pm 0,10)$ & $( \pm 0,04)$ \\
\hline \multirow{2}{*}{ Biyopsi } & 0,99 & 0,99 & 0,99 & 1,00 & 0,97 & 0,98 & 0,95 & 0,98 \\
& $( \pm 0,00)$ & $( \pm 0,00)$ & $( \pm 0,00)$ & $( \pm 0,00)$ & $( \pm 0,01)$ & $( \pm 0,03)$ & $( \pm 0,03)$ & $( \pm 0,01)$ \\
\hline
\end{tabular}

Aşırı rassal ağaçların eğitiminde rastgele ormanlar yönteminde kullanılan parametrelere benzer şekilde ağaç sayısı 10 olarak belirlenmiştir. Dügü̈mlerdeki bölünmenin kalitesini ölçmek için ise 'gini' fonksiyonu kullanılmıştır. Tablo 2'de aşırı rassal ağaçlardan 10-kat çapraz doğrulama ile elde edilen doğruluk, kesinlik, hassasiyet ve AUC performans ölçütlerinin ortalama ve standart sapmaları verilmiştir.

Tablo 2. Aşırı rassal ağaçlar algoritmasından elde edilen performans değerleri

\begin{tabular}{ccccccccc}
\hline & \multicolumn{4}{c}{ Ĕ̆itim } & \multicolumn{4}{c}{ Test } \\
\hline & Doğruluk & Kesinlik & Hassasiyet & AUC & Doğruluk & Kesinlik & Hassasiyet & AUC \\
\hline \multirow{2}{*}{ Hinselmann } & 1,00 & 0,99 & 1,00 & 1,00 & 0,99 & 0,99 & 1,00 & 1,00 \\
& $( \pm 0,00)$ & $( \pm 0,00)$ & $( \pm 0,00)$ & $( \pm 0,00)$ & $( \pm 0,01)$ & $( \pm 0,02)$ & $( \pm 0,00)$ & $( \pm 0,01)$ \\
\hline \multirow{2}{*}{ Schiller } & 1,00 & 0,99 & 1,00 & 1,00 & 0,96 & 0,97 & 0,95 & 0,98 \\
& $( \pm 0,00)$ & $( \pm 0,00)$ & $( \pm 0,00)$ & $( \pm 0,00)$ & $( \pm 0,02)$ & $( \pm 0,03)$ & $( \pm 0,03)$ & $( \pm 0,02)$ \\
\hline \multirow{2}{*}{ Sitoloji } & 1,00 & 0,99 & 0,99 & 1,00 & 0,97 & 0,94 & 0,84 & 0,92 \\
& $( \pm 0,00)$ & $( \pm 0,00)$ & $( \pm 0,00)$ & $( \pm 0,00)$ & $( \pm 0,02)$ & $( \pm 0,06)$ & $( \pm 0,10)$ & $( \pm 0,05)$ \\
\hline \multirow{2}{*}{ Biyopsi } & 1,00 & 0,99 & 1,00 & 1,00 & 0,97 & 0,97 & 0,95 & 0,98 \\
& $( \pm 0,00)$ & $( \pm 0,00)$ & $( \pm 0,00)$ & $( \pm 0,00)$ & $( \pm 0,01)$ & $( \pm 0,02)$ & $( \pm 0,04)$ & $( \pm 0,02)$ \\
\hline
\end{tabular}


Adaboost algoritması uygulanırken eğitimde temel öğrenici olarak karar ağacı kullanılmış ve eğitimin sonlanması için kullanılacak maksimum temel öğrenici sayısı 100 olarak belirlenmiştir. Bu parametreler kullanılarak Adaboost algoritmasıyla 10-kat çapraz doğrulama sonucunda bulunan sınıflama başarımına ait ortalama ve standart sapma değerleri Tablo 3’te gösterilmiştir.

Tablo 3. Adaboost yönteminin sınıflama başarımı

\begin{tabular}{ccccccccc}
\hline & \multicolumn{4}{c}{ Eğitim } & \multicolumn{4}{c}{ Test } \\
\hline & Doğruluk & Kesinlik & Hassasiyet & AUC & Doğruluk & Kesinlik & Hassasiyet & AUC \\
\hline \multirow{2}{*}{ Hinselmann } & 1,00 & 0,99 & 1,00 & 1,00 & 0,99 & 0,99 & 1,00 & 1,00 \\
& $( \pm 0,01)$ & $( \pm 0,01)$ & $( \pm 0,01)$ & $( \pm 0,00)$ & $( \pm 0,04)$ & $( \pm 0,05)$ & $( \pm 0,01)$ & $( \pm 0,02)$ \\
\hline \multirow{2}{*}{ Schiller } & 1,00 & 0,99 & 1,00 & 1,00 & 0,96 & 0,97 & 0,95 & 0,98 \\
& $( \pm 0,02)$ & $( \pm 0,01)$ & $( \pm 0,04)$ & $( \pm 0,00)$ & $( \pm 0,03)$ & $( \pm 0,05)$ & $( \pm 0,04)$ & $( \pm 0,03)$ \\
\hline \multirow{2}{*}{ Sitoloji } & 1,00 & 0,99 & 0,99 & 1,00 & 0,97 & 0,94 & 0,84 & 0,92 \\
& $( \pm 0,01)$ & $( \pm 0,01)$ & $( \pm 0,04)$ & $( \pm 0,00)$ & $( \pm 0,02)$ & $( \pm 0,11)$ & $( \pm 0,08)$ & $( \pm 0,07)$ \\
\hline \multirow{2}{*}{ Biyopsi } & 1,00 & 0,99 & 1,00 & 1,00 & 0,97 & 0,97 & 0,95 & 0,98 \\
& $( \pm 0,01)$ & $( \pm 0,01)$ & $( \pm 0,04)$ & $( \pm 0,00)$ & $( \pm 0,02)$ & $( \pm 0,06)$ & $( \pm 0,04)$ & $( \pm 0,03)$ \\
\hline
\end{tabular}

Çalışmada kullanılan kolektif öğrenme yöntemlerinin performansı 10-kat çapraz doğrulama ile test verilerinden elde edilen doğruluk ölçütüne göre Şekil 1'de verilen grafik ile karşılaştırılmıştır.

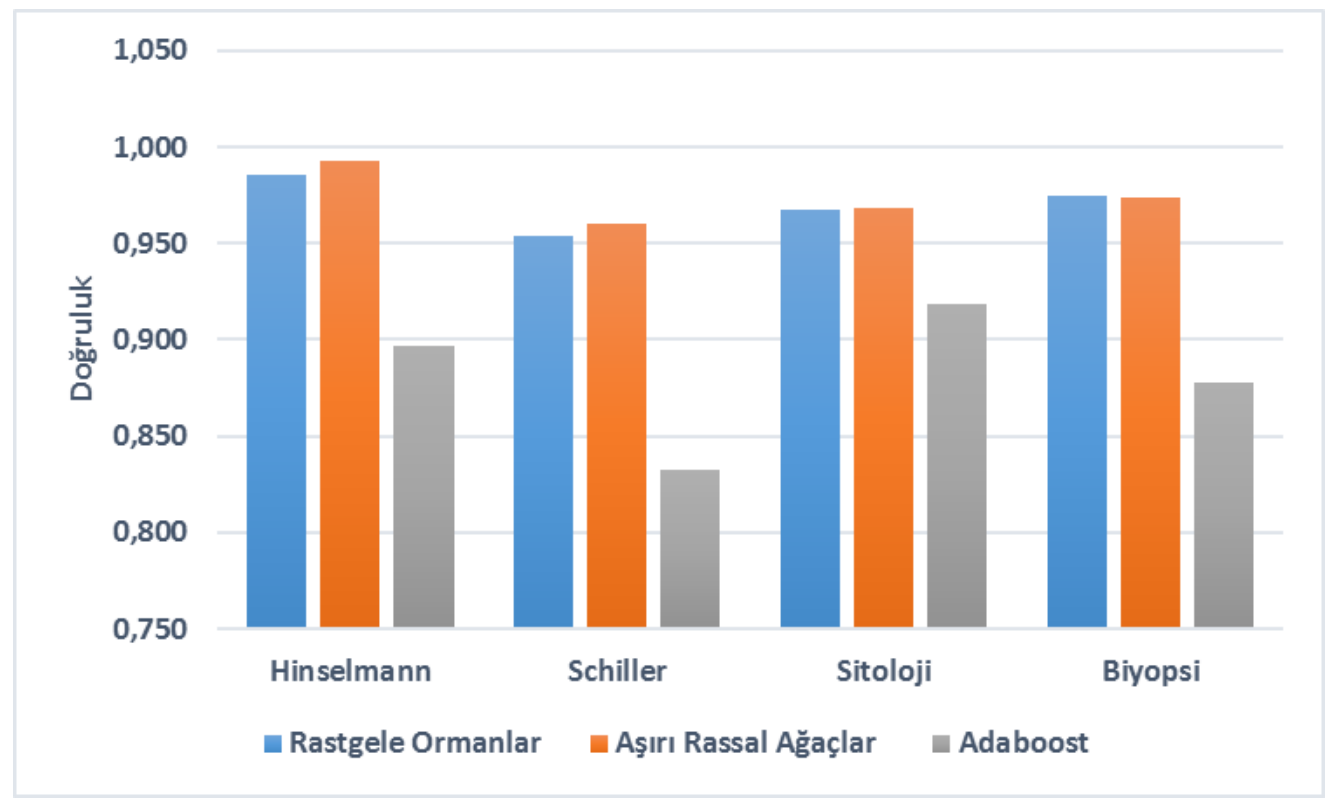

Şekil 1. Test verileri üzerinde kolektif öğrenme algoritmalarının başarımı

$\mathrm{Bu}$ çalışmadan elde edilen sonuçlar aynı veri setini kullanarak yapılmış ve serviks kanseri riskini 4 farklı tarama yöntemi açısından sınıflandıran literatürdeki tek çalışma olan Wu ve Zhou'nun [18] çalışması ile kıyaslanmıştır. Tablo 4 'te bu çalışmadaki en yüksek performansı gösteren aşırı rassal ağaçlar yöntemi ile elde edilen doğruluk sonuçları, Wu ve Zhou'nun [18] çalışmasında kullandıkları destek vektör 
makineleri (DVM), özyinelemeli eleme yöntemini kullanan DVM (DVM-RFE) ve temel bileşen analizi yöntemini kullanan DVM (DVM-PCA) yöntemleri ile karşılaştırılmıştır. Tablo4'teki değerlerden de görülebileceği gibi bu çalışmada kullanılan aşırı rassal ağaçlar yöntemi DVM yöntemi ve onun türevlerine göre 4 farklı tarama türünün hepsi için serviks kanseri riskini belirlemede daha yüksek başarıma sahiptir.

Tablo 4. Bulguların diğer çalışmalarla kıyaslanması

\begin{tabular}{ccccc}
\hline & Hinselmann & Schiller & Sitoloji & Biyopsi \\
\hline $\begin{array}{c}\text { Aşırı rassal ağaçlar } \\
\text { (Bu çalışma) }\end{array}$ & $\% 99,30$ & $\% 96,00$ & $\% 96,86$ & $\% 97,33$ \\
\hline $\begin{array}{c}\text { DVM } \\
{[\mathbf{1 8}]}\end{array}$ & $\% 93,97$ & $\% 90,18$ & $\% 92,75$ & $\% 94,13$ \\
\hline $\begin{array}{c}\text { DVM-RFE } \\
{[\mathbf{1 8}]}\end{array}$ & $\% 93,69$ & $\% 90,18$ & $\% 92,37$ & $\% 94,03$ \\
\hline $\begin{array}{c}\text { DVM-PCA } \\
{[\mathbf{1 8}]}\end{array}$ & $\% 93,79$ & $\% 90,18$ & $\% 92,46$ & $\% 94,03$ \\
\hline
\end{tabular}

\section{SONUC}

$\mathrm{Bu}$ çalışmada çeşitli risk faktörleri kullanılarak 4 farklı tarama tekniği açısından hastaların serviks kanseri riski taşıyıp taşımadığı tahmin edilmiştir. Kolektif öğrenme metotlarından rastgele ormanlar, aşırı rassal ağaçlar ve Adaboost algoritmaları bu amaçla 10-kat çapraz doğrulama yöntemiyle eğitilmiştir. Çalışmadan elde edilen ikili sınıflandırma sonuçlarına göre rastgele ormanlar ve aşırı rassal ağaçlar algoritmaları bir birine çok yakın sınıflama performansı göstermiştir. Bununla birlikte aşırı rassal ağaçların başarımının Hinselmann ve Schiller yöntemleri için biraz daha yüksek olduğu görülmüştür. Bu çalışmada Adaboost algoritması en düşük sınıflama başarısına sahip yöntem olmuştur. Aşırı rassal ağaçlar algoritmasından Hinselmann, Schiller, sitoloji ve biyopsi tarama yöntemleri için hastaların serviks kanseri olması riskinin tahmin başarısı sırasıyla $\% 99, \% 96, \% 97$ ve $\% 97$ olmuştur. $\mathrm{Bu}$ doğruluk oranları sınıflandırma kesinliği ve hassasiyetinden elde edilen yüksek performans ile birlikte değerlendirildiğinde aşırı rassal ağaçlar yönteminin hastaların serviks kanseri riskinin değerlendirilmesinde doktorlara yardımcı bir KKDS olarak kullanabileceğini göstermiştir.

\section{KAYNAKLAR}

[1] M.A. Musen, B. Middleton, R.A. Greenes, "Clinical Decision-Support Systems," Biomedical Informatics, 4. bask1. London: Springer, 2014, böl. 20, ss. 643-674.

[2] J.M. Hardin ve D.C. Chhieng, "Data Mining and Clinical Decision Support Systems," Clinical decision support systems, 2. bask1. New York: Springer Science+ Business Media, LLC, 2007, böl. 3 , ss. 44-63. 
[3] N. Ye, C. Liu, P. Shi, "Metabolomics Analysis of Cervical Cancer, Cervical Intraepithelial Neoplasia and Chronic Cervicitis by 1H NMR Spectroscopy," European Journal of Gynaecological Oncology, c. 36, s. 2, ss. 174-180, 2015.

[4] K. Fernandes, J.S. Cardoso, J. Fernandes, "Transfer Learning with Partial Observability Applied to Cervical Cancer Screening," ${ }^{\text {th }}$ Iberian Conference on Pattern Recognition and Image Analysis, Faro, Portugal, 2017, ss. 243-250.

[5] A. Bandyopadhyay, U. Mukherjee, S. Ghosh, S. Ghosh, S.K. Sarkar, "Pattern of Failure with Locally Advanced Cervical Cancer- A Retrospective Audit and Analysis of Contributory Factors," Asian Pacific Journal of Cancer Prevention, c. 19, s. 1, ss. 73-79, 2017.

[6] W.A. Leyden, M.M Manos, A.M. Geiger, S. Weinmann, J. Mouchawar, K. Bischoff, M.U. Yood, J. Gilbert, S.H. Taplin, "Cervical Cancer in Women with Comprehensive Health Care Access: Attributable Factors in the Screening Process," Journal of the National Cancer Institute, c. 97, s. 9, ss. 675-683, 2005.

[7] P. Bountris, M. Haritou, A. Pouliakis, N. Margari, M. Kyrgiou, A. Spathis, A. Pappas, I. Panayiotides, E.A. Paraskevaidis, P. Karakitsos, D.D. Koutsouris, "An intelligent clinical decision support system for patient-specific predictions to improve cervical intraepithelial neoplasia detection," BioMed Research International, c. 2014, Article Number: 341483, 2014.

[8] A. Demirhan, "Neuroimage-based clinical prediction using machine learning tools," International Journal of Imaging Systems and Technology, c. 27, s. 1, ss. 89-97, 2017.

[9] N.A. Mayr, W.T. Yuh, J. Zheng, J.C. Ehrhardt, V.A. Magnotta, J.I. Sorosky, R.E. Pelsang, L.W. Oberley, D.H. Hussey, "Prediction of tumor control in patients with cervical cancer: analysis of combined volume and dynamic enhancement pattern by MR imaging," American Journal of Roentgenology, c. 170, s. 1, ss. 177-182, 1998.

[10] M. Galar, A. Fernandez, E. Barrenechea, H. Bustince, F. Herrera, "A review on ensembles for the class imbalance problem: bagging-, boosting-, and hybrid-based approaches," IEEE Transactions on Systems, Man, and Cybernetics, Part C (Applications and Reviews), c. 42, s. 4, ss. 463-484, 2012.

[11] P. Bühlmann, "Bagging, Boosting and Ensemble Methods," Handbook of Computational Statistics, 2. bask1. Berlin: Springer-Verlag, 2012, ss. 985-1022.

[12] L. Breiman, "Random Forests,” Machine Learning, c. 45, s. 1, ss. 5-32, 2001.

[13] P. Geurts, D. Ernst, L. Wehenkel, "Extremely randomized trees," Machine Learning, c. 63, s. 1, ss. 3-42, 2006.

[14] Y. Freund, R.E. Schapire, "A Decision-Theoretic Generalization of On-Line Learning and an Application to Boosting," Journal of Computer and System Sciences, c. 55, s. 1, ss. 119-139, 1997.

[15] T. Hastie, S. Rosset, J. Zhu, H. Zou, "Multi-Class Adaboost," Statistics and its Interface, c. 2, s. 3, ss. 349-360, 2009. 
[16] T. Hastie, R. Tibshirani, J. Friedman, The Elements of Statistical Learning, Second Edition, New York: Springer series in statistics, 2009, böl. 10, ss. 337-384.

[17] F. Pedregosa, G. Varoquaux, A. Gramfort, V. Michel, B. Thirion, O. Grisel, M. Blondel, P. Prettenhofer, R. Weiss, V. Dubourg, J. Vanderplas, A. Passos, D. Cournapeau, M. Brucher, M. Perrot, É. Duchesnay, "Scikit-learn: Machine learning in Python," Journal of Machine Learning Research, c. 12, s. Oct, ss. 2825-2830, 2011.

[18] W. Wu, H. Zhou, "Data-Driven Diagnosis of Cervical Cancer with SVM-Based Approaches," IEEE Access, c. 5, ss. 25189-25195, 2017. 